\title{
28 Research Soure \\ Frequency of Tooth Brushing as a Predictive Factor for Future Kidney Function Decline
}

\section{Keita Hirano ( $\nabla$ keita@kuhp.kyoto-u.ac.jp )}

St Luke's International Hospital https://orcid.org/0000-0003-3695-9056

\section{Takuro Shimbo}

Ohta Nishinouchi Hospital

\section{Yasuhiro Komatsu}

Gunma University Graduate School of Medicine

\section{Daiki Kobayashi}

St Luke's International Hospital

\section{Original Article}

Keywords: Chronic kidney disease, Kidney function, Oral and periodontal health, Tooth brushing frequency

Posted Date: February 16th, 2021

DOI: https://doi.org/10.21203/rs.3.rs-164266/v1

License: (1) (1) This work is licensed under a Creative Commons Attribution 4.0 International License. Read Full License

Version of Record: A version of this preprint was published at Journal of Nephrology on February 22nd, 2021. See the published version at https://doi.org/10.1007/s40620-021-00987-2. 


\section{Abstract}

\section{Background}

Tooth brushing is important for maintaining oral health and preventing periodontal diseases, which commonly arise in patients with chronic kidney disease (CKD). However, the association between tooth brushing frequency and kidney function decline remains unclear.

\section{Methods}

We conducted a retrospective longitudinal study at St Luke's International Hospital, Japan, and participants who underwent health examinations at the Centre for Preventive Medicine from 2005 to 2011 were included. Participants' tooth brushing frequencies were assessed; multivariate analyses were conducted using a generalized estimating equation to evaluate the association between tooth brushing frequency and a composite renal outcome - composed of a $25 \%$ eGFR reduction, an eGFR of $<15$ $\mathrm{mL} / \mathrm{min} / 1.73 \mathrm{~m}^{2}$, and a requirement for regular dialysis - after adjusting for potential covariates. We also stratified participants by baseline CKD risk category to perform sub-analyses.

\section{Results}

Overall, 76,472 participants were included (mean age of 45.9 years) and 38,233 (50\%) were male. During follow-up, 8,219 participants (10.8\%) experienced composite renal outcomes. Brushing teeth at least once to twice a day was associated with significantly lower incidences of composite renal outcomes than brushing teeth less frequently (adjusted odds ratio [OR]: 0.26 ; $95 \%$ confidence interval [CI]: $0.24-0.28$ for once to twice a day; adjusted OR: $0.65 ; 95 \% \mathrm{Cl}: 0.62-0.69$ for after every meal). In our sub-analyses, brushing at least once to twice a day related to a decreased likelihood of composite renal outcomes; however, this effect was only observed within the low and moderate baseline risk groups.

\section{Conclusion}

Frequent tooth brushing benefits oral health and may prevent kidney function decline, which could have implications for other systemic diseases. However, a longitudinal cohort study is required to confirm whether tooth brushing can prevent poor renal outcomes.

\section{Introduction}

Over the last decade, chronic kidney disease (CKD) has become a leading public health issue worldwide [1]. Individuals with CKD have a higher mortality rate than the comparable healthy population, especially those with advanced CKD requiring renal replacement therapy [2]. Furthermore, renal replacement therapy leads to an increased burden of medical expenses on patients.

Good oral hygiene, in which tooth brushing plays a major role, is clearly associated with periodontal disease (PD) [3], and a reduced risk of cancer and cardiovascular events owing to its ability to prevent PD. 
$[4,5]$. Moreover, robust evidence shows several factors such as smoking, diabetes, medications, obesity and adverse pregnancy outcomes identified as a risk factors for the development of periodontitis [6]. To prevent PD, National Institute of Dental and Craniofacial Research recommends brushing teeth twice a day with a fluoride toothpaste. PD is a common group of inflammatory diseases affecting about half of the global adult population, which can eventually lead to teeth loss. Notably, previous studies have suggested that poor periodontal health is more prevalent and severe in adults with CKD [7]. The relationship between PD and kidney function decline has been extensively studied in the elderly population in particular [8].

Despite the evident connection between PD and CKD, studies on the relationship between these two conditions have been lacking as they have only offered imprecise and inconsistent risk estimates [9-11]. Grubbs et al. performed a retrospective study with 761 elderly men, reporting that severe PD was significantly associated with kidney function decline, even after adjusting for age and well-known CKD risk factors such as smoking status and history of diabetes mellitus or hypertension [12]. In 2018, metaanalyses indicated an association between CKD and PD. The strength of this association increased when severe PD was specifically considered [13]; however, the effects of tooth brushing on kidney function decline in the general population have not yet been definitively demonstrated. Accordingly, studies are necessary to determine whether preventing or treating poor oral health can be related to a decrease in the incidence and/or severity of CKD.

To address the shortcomings of previous studies, we conducted a longitudinal cohort study to elucidate the relationship between tooth brushing and kidney function in healthy individuals. To do so, we evaluated the association between tooth brushing and kidney function decline among a general population cohort over approximately 10 years. We hypothesized that individuals who practice less frequent tooth brushing would experience greater kidney function decline.

\section{Methods}

A retrospective longitudinal cohort study was conducted at St Luke's International Hospital, a large teaching hospital in Tokyo, Japan. All participants who underwent health examinations at the hospital's Centre for Preventive Medicine between 2005 and 2011 and had an estimated glomerular filtration rate (eGFR) $>15 \mathrm{~mL} / \mathrm{min} / 1.73 \mathrm{~m}^{2}$ were included. Participants who required regular dialysis, regardless of whether it was hemo- or peritoneal dialysis, were excluded. The primary outcome was a composite renal outcome that was compared with the frequency of tooth brushing. The St Luke's Ethics Committee Institutional Review Board approved this study (approval number: 18-R203 - Comprehensive approvals for studies about social habits using this dataset).

\section{Outcomes}

The primary outcome was a composite renal outcome consisting of a $25 \%$ eGFR reduction from baseline, an eGFR of $<15 \mathrm{~mL} / \mathrm{min} / 1.73 \mathrm{~m}^{2}$, and a requirement for regular dialysis [14-16]. The eGFR for each patient was calculated based on the following formula for Japanese patients [17]: 
Male: eGFR $=194 \times(\text { serum creatinine })^{-1.094} \times(\text { age })^{-0.287}$

Female: eGFR $=194 \times(\text { serum creatinine })^{-1.094} \times(\text { age })^{-0.287} \times 0.739$

The baseline eGFR for each participant was defined as the eGFR measured at their first visit to the center between 2005 and 2011. The eGFR was measured at every subsequent visit to the center for health examinations. The initiation of regular dialysis was dependent on each physician's decision; relevant information was obtained from the electronic medical records or participants' self-reports. Each patient was followed until December 31, 2018.

\section{Frequency of tooth brushing}

All participants were asked about their tooth brushing frequency as part of a questionnaire during their health examinations between 2005 and 2011. Each participant was asked to select one of the following responses to the question regarding tooth brushing frequency: less than daily, once a day, once to twice a day, or after every meal. Due to the small number of participants who brushed their teeth less than daily, we combined this group of patients with those that brushed their teeth once a day; this was defined as the reference group. Information about tooth brushing frequency was subsequently obtained at every visit to the center and considered as a time-dependent variable.

\section{Covariates}

As a part of the questionnaire provided during health examinations, we obtained information about participants' demographics, medical histories, laboratory measures and physical examinations as potential covariates for our study. Health habits including alcohol consumption (abstainer, occasional drinker, regular drinker), smoking status (never smoker, former smoker, current smoker) and exercise habits (almost none, 1-2 times a week, 3-5 times a week, almost every day) were also assessed. During the health examinations, trained staff measured participants' heights and weights and calculated their body mass indexes (BMI). Each participant was categorized into one of three groups based on their BMI and Asian criteria: underweight (BMI $<18.5 \mathrm{~kg} / \mathrm{m}^{2}$ ), normal (BMI $18.5-24.9 \mathrm{~kg} / \mathrm{m}^{2}$ ) or obese/overweight $\left(\mathrm{BMI} \geq 25.0 \mathrm{~kg} / \mathrm{m}^{2}\right)$ [18]. Information about comorbidities related to the progression of CKD such as diabetes, hypertension [19], or cancer [20], was also obtained from participants' self-reports. This information was obtained at every health examination and considered as a time-dependent variable.

Finally, information regarding participants' baseline kidney function was also evaluated and retained as a potential covariate. The eGFR of each participant was calculated based on the Japanese formula outlined above and used to categorize patients into a baseline CKD risk group (low risk, moderately increased risk, high risk, very high risk) according to The Kidney Disease: Improving Global Outcomes (KDIGO) 2012 Clinical Practice Guideline [21]. The baseline eGFRs and CKD risk group classifications were used as covariates.

\section{Statistical methods}


First, we compared participants' baseline characteristics with their tooth brushing frequency. We then conducted multivariate analyses using a generalized estimating equation with the logit function and a binomial distribution to evaluate the association between frequency of tooth brushing and composite renal outcome, adjusting for potential covariates. We used different potential covariates in each of the models: Model 1 was adjusted for participant age and sex, baseline eGFR, and baseline CKD risk category; Model 2 was adjusted for health habits (alcohol consumption, smoking status, and exercise habits) in addition to the covariates used in Model 1; Model 3 was adjusted for BMI in addition to the covariates used in Model 2; and Model 4 was adjusted for comorbidities (hypertension, diabetes, and any type of cancer) in addition to the covariates used in Model 3. Our final model was model 4, but we also presented the results of other models as part of the sensitivity analysis to show that the results are consistent with varying covariates. We also stratified the data by baseline CKD risk category to evaluate the impact of tooth brushing frequency on composite renal outcomes in sub-analyses. All analyses were performed using STATA 14 (STATA Corp., College Station, TX, USA).

\section{Results}

A total of 76,472 participants were included in this study. The mean age of the population was $45.9 \pm$ 12.4 years, and 38,233 participants $(50 \%)$ were male. Table 1 summarizes participants' baseline characteristics categorized by tooth brushing frequency. Participants who brushed their teeth after every meal tended to be younger and female and also exhibited healthier habits, such as drinking less regularly and exercising more, fewer were current smokers, and they had a lower BMI on average than those who brushed their teeth less often.

The findings regarding comorbidities were inconsistent across groups divided by tooth brushing frequency. Those who brushed their teeth once to twice a day had a lower prevalence of cancer and diabetes, whereas those who brushed their teeth after every meal exhibited a lower prevalence of hypertension. In terms of kidney function, those who brushed their teeth at least once to twice a day had a higher eGFR and milder stage of CKD than those who brushed their teeth once a day or less. However, those who brushed their teeth after every meal tended to belong to a more severe CKD risk category, suggesting more severe proteinuria, than those who brushed their teeth once to twice a day.

During the median follow-up period of 68.3 months (Interquartile range: $26.1-117.6$ months), 8,219 (10.8\%) patients experienced composite renal outcomes. Tooth brushing after every meal (adjusted odds ratio [OR]: $0.65 ; 95 \%$ confidence interval [Cl]: $0.62-0.69$ ) or once to twice a day (adjusted OR: $0.26 ; 95 \% \mathrm{Cl}$ : 0.24-0.28) was significantly associated with a lower incidence of composite renal outcomes compared to brushing teeth once a day or less according to the analysis by Model 4 (Table 2). In our sub-analyses stratified by CKD risk category, tooth brushing once to twice a day or after every meal was associated with a lower frequency of composite renal outcomes among those who belonged to the low baseline CKD risk group (adjusted OR: $0.24,0.66 ; 95 \% \mathrm{Cl}: 0.23-0.27,0.62-0.70$, respectively) or the moderate baseline CKD risk group (adjusted OR: 0.34, 0.45; 95\% Cl: 0.25-0.45, 0.34-0.60, respectively). However, this 
positive relationship between tooth brushing and renal outcomes was not observed among participants who belonged to the high or very high baseline CKD risk groups (Table 3).

\section{Discussion}

This study indicates that good oral health practices may be related to a decrease in the risk of kidney function decline in a healthy population undergoing yearly medical examinations, independent of additional risk factors such as other health habits and comorbidities. Frequent tooth brushing was associated with a decreased risk of composite renal outcomes defined by a $25 \%$ eGFR reduction from baseline values, an eGFR of $<15 \mathrm{~mL} / \mathrm{min} / 1.73 \mathrm{~m}^{2}$ (stage $5 \mathrm{CKD}$ ), and a requirement for regular dialysis. Notably, those who brushed their teeth once to twice a day exhibited an elevated eGFR and milder CKD staging compared to those who brushed their teeth after every meal. A previous study revealed that a high concentration of salivary urea is associated with a bitter taste in patients with CKD [22], and patients with CKD possibly brush their teeth more frequently because of the bitter taste, whereas our data show that there was no large difference in the severity of CKD among the different groups classified according to the frequency of tooth brushing. In sub-analyses stratified by baseline CKD risk group, frequent tooth brushing was associated with a lower frequency of composite renal outcomes in the low and moderate risk groups. Interestingly, however, frequent tooth brushing was not associated with a lower frequency of composite renal outcomes in the high or very high CKD risk groups.

Individuals with CKD may exhibit an increased prevalence of oral disease due to the interrelation of CKD with inflammation and malnutrition, leaving adults with CKD more prone to PDs [23]. According to a recent systematic review, poor oral health is highly prevalent in adults with CKD and the average severity of oral disease is increased in this population [24]. Furthermore, in adults treated with hemodialysis, poor dental health was associated with early mortality, whereas preventative oral care practices were associated with longer survival [25]. While a considerable amount of information about oral disease as a determinant of health in individuals with CKD is available, the link between oral health and kidney function decline is less clear because such studies were limited to PD and provided only imprecise risk estimates. A previous study reported that a low frequency of tooth brushing was associated with risk factors for CKD such as diabetes [26], whereas poor oral health was associated with renal impairment [27]. Another study indicated that clinically successful, non-surgical periodontal treatment both improved glycemic control and reduced the levels of renal impairment biomarkers and albumin in the urine $[28,29]$. Some studies have also indicated that periodontal treatment results in a significant improvement in median kidney function, as well as a reduction in the level of endothelial damage biomarkers, in patients with severe chronic periodontitis [30].

There are two possible explanations as to why a low frequency of tooth brushing is associated with renal impairment. The first explanation is that a low frequency of tooth brushing can lead to poor oral health, the result of which is an increase in the prevalence of PD. As PD is a known risk factor for CKD risk 
factors such as diabetes and cardiovascular disease, poor oral health can therefore lead to adverse outcomes in CKD mediated by endothelial dysfunction, atherosclerosis, thrombosis and chronic inflammation [31]. The second explanation is that PD directly causes alterations in the renal tissue. In animal models, induced periodontitis caused morphological changes in the renal tissue, as well as disruption of the brush border in the renal tubules. These alterations are both associated with increased oxidative stress within the kidneys [32,33]. Furthermore, the progression of periodontitis is associated with poor prognostic factors for CKD patients because the progression of periodontitis is implicated in malnutrition and protein-energy wasting syndrome [24]. Thus, PD, which can be mitigated by regular tooth brushing, may be a predictive factor for progressive kidney disease [34, 35].

Interestingly, we found that tooth brushing once to twice a day was associated with better kidney function than tooth brushing after every meal. The precise reason for this effect is unclear; however, there are some possible contributing factors. Generally, more frequent tooth brushing is preferred for the effective maintenance of good oral health and prevention of PD. However, one study reported that there is a tendency for an increased incidence of dental caries among patients who brush their teeth 3 times daily or more compared to those who brush their teeth twice daily or less [36]. Excessive tooth brushing can also potentially cause dental erosion; thus, patients should reduce the frequency of tooth brushing to avoid damaging the acquired pellicle [37]. Tooth brushing immediately after eating should particularly be avoided as acidic food or drink may weaken tooth enamel, which can result in enamel removal after brushing. Based on these factors, tooth brushing after every meal is likely excessive and may be counterproductive to maintaining good oral hygiene and preventing PD. This, in turn, may contribute to the kidney function decline observed in the patient group brushing after every meal compared to the group brushing once to twice a day.

With regards to our stratified sub-analyses by CKD risk category, we observed that brushing once to twice a day related to a decreased likelihood of composite renal outcomes in the low and moderate baseline risk groups. However, the positive effect of tooth brushing was not observed in the high and very high baseline risk groups. We believe that this may be attributable to an irreversible disease stage in the highrisk group, past which point preventative methods will not impact renal outcomes unless the preventative action is directly targeting a major risk factor. Nonetheless, regular tooth brushing is an inexpensive intervention that poses no risk of complications; therefore, more frequent daily tooth brushing, not overbrushing, should be recommended to potentially mitigate further renal impairment.

This study has several limitations. First, there is a possible residual selection bias as all participants were from a single center. The dataset we used consisted of data obtained from patients who visited for health check-up, which is mainly voluntary, and those who visited to undergo screening examinations for chronic diseases and cancer. A previous study revealed that socio-cultural differences influenced oral health and dental disease [38]; therefore, it is possible that we are dealing with a group of patients who are more health conscious than the general population. The number of patients assigned to the three groups categorized according to the frequency of tooth brushing is not equal; however, this large-scale cohort study provides a high-quality medical record database due to the low exclusion rate, which may mitigate 
the potential bias as all data were well controlled and reported. Second, there may be some overlap in each group. For example, once a day may be classified as "once a day or less" or "once or twice a day". However, because frequency of brushing tooth can fluctuate in our long-term retrospective cohort study of 10 years or more, the data at the first visit does not have a significant meaning in itself. On the other hand, GEE we used in this study is more accurate than simple logistic regression because it enabled the data of the annual tooth brushing frequency as a time-dependent variable." Third, we could not assess or adjust data according to access to dental care, exposure to fluoridation, or the use of dental floss and interdental cleansers, among other factors. It may be affected by the frequency of brushing teeth and cultural and social levels; however, additional information on school education, work or income and has not been measured in this retrospective study. Nevertheless, we strictly considered several possible confounding factors such as age, sex, baseline eGFR, health habits, BMI, and comorbidities. Finally, a $25 \%$ eGFR reduction was used as a factor in the renal composite outcome. However, a $25 \%$ eGFR reduction can also be observed with acute renal injury or normal variability (i.e. random error). To account for this possibility, we integrated an eGFR of $<15 \mathrm{~mL} / \mathrm{min} / 1.73 \mathrm{~m}^{2}$ and dialysis initiation into the composite renal outcome for our large-scale, long-term study. The number of enrolled patients with CKD stage 4, having GFR values close to $15 \mathrm{ml} / \mathrm{min} / \mathrm{min} / 1.73 \mathrm{~m}^{2}$, was 31 , accounting for only $0.04 \%$ of all participants. And also, the subgroup analysis of CKD risk categories has been shown to be associated with tooth brushing frequency and renal composite outcome even in the low risk group shown by Table 4 . (Table 4 is not available in this version.) Therefore, we think that our study evaluated a possible true association between kidney function decline and tooth brushing frequency.

In conclusion, our study demonstrated that a low frequency of tooth brushing was associated with poorer composite renal outcomes over time; however, a longitudinal cohort study is required to confirm whether tooth brushing can prevent poor renal outcomes. Thus, once to twice a day tooth brushing practices may have a positive impact on preventing CKD, which could have implications for other systemic diseases.

\section{Declarations}

Funding: This study was not supported by external funding.

Conflicts of Interest: The authors have no conflicts of interest to declare.

Ethics Approval: The St Luke's Ethics Committee Institutional Review Board approved this study (approval number: 18-R203 - Comprehensive approvals for studies about social habits using this dataset).

Consent to Participate: The requirement for informed consent was waived due to the retrospective nature of this study.

Consent for Publication: The requirement for informed consent was waived due to the retrospective nature of this study.

Availability of Data and Material: Not applicable 
Code Availability: Not applicable

Author Contributions: K.H. conceived the ideas of the study and designed and performed the study. K.H. and D.K. analyzed the data, performed statistical analysis, and wrote the paper. T.S., Y.K. and D.K. participated in the study design and coordination. All authors discussed the results and implications, commented on the manuscript at all stages, and approved the final manuscript.

\section{References}

1. Gansevoort RT, Correa-Rotter R, Hemmelgarn BR et al (2013) Chronic kidney disease and cardiovascular risk: epidemiology, mechanisms, and prevention. Lancet 382:339-352. https://doi.org/10.1016/S0140-6736(13)60595-4

2. Mailloux LU, Bellucci AG, Wilkes BM et al (1991) Mortality in dialysis patients: analysis of the causes of death. Am J Kidney Dis 8:326-335.

3. Zimmermann H, Zimmermann N, Hagenfeld D, Veile A, Kim TS, Becher H (2015) Is frequency of tooth brushing a risk factor for periodontitis? A systematic review and meta-analysis. Community Dent Oral Epidemiol 43:116-127. https://doi.org/10.1111/cdoe.12126

4. Kobayashi D, Takahashi O, Shimbo T (2019) Frequency of Daily Tooth Brushing and Development of any Type of Malignancy. Anticancer Res 39:4415-4421. https://doi.org/10.21873/anticanres.13613

5. Kobayashi D, Mizuno A, Mitsui R, Shimbo T (2020) Frequency of daily tooth brushing and subsequent cardiovascular events. Coron Artery Dis 31:545-549. https://doi.org/10.1097/MCA.0000000000000882.

6. Nazir MA (2017) Prevalence of periodontal disease, its association with systemic diseases and prevention. Int J Health Sci (Qassim) 11:72-80.

7. Chambrone L, Foz AM, Guglielmetti MR et al (2013) Periodontitis and chronic kidney disease: a systematic review of the association of diseases and the effect of periodontal treatment on estimated glomerular filtration rate. J Clin Periodontol 40:443-456. https://doi.org/10.1111/jcpe.12067

8. Iwasaki M, Taylor GW, Nesse W, Vissink A, Yoshihara A, Miyazaki H (2012) Periodontal disease and decreased kidney function in Japanese elderly. Am J Kidney Dis 59:202-209. https://doi.org/10.1053/j.ajkd.2011.08.027

9. Fisher MA, Taylor GW, Shelton BJ et al (2008) Periodontal disease and other nontraditional risk factors for CKD. Am J Kidney Dis 51:45-52. https://doi.org/10.1053/j.ajkd.2007.09.018

10. Schütz JDS, de Azambuja CB, Cunha GR, et al (2020) Association between severe periodontitis and chronic kidney disease severity in predialytic patients: A cross-sectional study. Oral Dis 26:447-456. doi: 10.1111/odi.13236

11. Dannewitz B, Sommerer C, Stölzel P, et al (2020) Status of periodontal health in German patients suffering from chronic kidney disease-Data from the GCKD study. J Clin Periodontol 47:19-29. doi: $10.1111 /$ jcpe. 13208 
12. Grubbs V, Vittinghoff E, Taylor G et al (2016) The association of periodontal disease with kidney function decline: a longitudinal retrospective analysis of the MrOS dental study. Nephro Dial Transpl 31:466-472. https://doi.org/10.1093/ndt/gfv312

13. Deschamps-Lenhardt S, Martin-Cabezas R, Hannedouche T, Huck O (2019) Association between periodontitis and chronic kidney disease: Systematic review and meta-analysis. Oral Dis 25:385402. https://doi.org/10.1111/odi.12834

14. Hung AM, Roumie CL, Greevy RA et al (2013) Kidney function decline in metformin versus sulfonylurea initiators: assessment of time-dependent contribution of weight, blood pressure, and glycemic control. Pharmacoepidem Dr S 22:623-631. https://doi.org/10.1002/pds.3432

15. Ricardo AC, Yang W, Sha D et al (2019) Sex-Related Disparities in CKD Progression. J Am Soc Nephro 30:137-146. https://doi.org/10.1681/ASN.2018030296

16. Hung AM, Roumie CL, Greevy RA et al (2012) Comparative effectiveness of incident oral antidiabetic drugs on kidney function. Kidney Int 81:698-706. https://doi.org/10.1038/ki.2011.444

17. Matsuo S, Imai E, Horio M et al (2009) Revised equations for estimated GFR from serum creatinine in Japan. Am J Kidney Dis 53:982-992. https://doi.org/10.1053/j.ajkd.2008.12.034

18. WHO Expert Consultation (2004) Appropriate body-mass index for Asian populations and its implications for policy and intervention strategies. Lancet 363:157-163. https://doi.org/10.1016/S0140-6736(03)15268-3

19. Levey AS, Atkins R, Coresh J et al (2007) Chronic kidney disease as a global public health problem: Approaches and initiatives - a position statement from Kidney Disease Improving Global Outcomes. Kidney Int 72:247-259.

20. Humphrey LL, Fu R, Buckley DI, Freeman M, Helfand M (2008) Periodontal disease and coronary heart disease incidence: a systematic review and meta-analysis. J Gen Intern Med 23: 2079-2086. https://doi.org/10.1007/s11606-008-0787-6

21. KDIGO (2013). Clinical practice guideline for the evaluation and management of chronic kidney disease.

22. Brennan F, Stevenson J, Brown M (2020) The Pathophysiology and Management of Taste Changes in Chronic Kidney Disease: A Review. J Ren Nutr 30:368-379. doi: 10.1053/j.jrn.2019.11.004

23. Dande R, Gadbail AR, Sarode $S$ et al (2018) Oral manifestations in diabetic and nondiabetic chronic renal failure patients receiving hemodialysis. J Contemp Dent Prac 19:398-403. https://doi.org/10.5005/jp-journals-10024-2273

24. Ruospo M, Palmer SC, Craig JC et al (2014) Prevalence and severity of oral disease in adults with chronic kidney disease: a systematic review of observational studies. Nephro Dial Transl 29:364375. https://doi.org/10.1093/ndt/gft401

25. Palmer SC, Ruospo M, Wong G, et al (2015) Dental Health and Mortality in People With End-Stage Kidney Disease Treated With Hemodialysis: A Multinational Cohort Study. Am J Kidney Dis 66:666676. doi: 10.1053/j.ajkd.2015.04.051 
26. Kuwabara M, Motoki Y, Ichiura K et al (2016) Association between toothbrushing and risk factors for cardiovascular disease: a large-scale, cross-sectional Japanese study. BMJ Open 6:e009870. https://doi.org/10.1136/bmjopen-2015-009870

27. Ní Chróinín D, Montalto A, Jahromi S, Ingham N, Beveridge A, Foltyn P (2016) Oral Health Status Is Associated with Common Medical Comorbidities in Older Hospital Inpatients. J Am Geriatr Soc 64:1696-1700. https://doi.org/10.1111/jgs.14247

28. Hayashi J, Hasegawa A, Hayashi K et al (2017) Effects of periodontal treatment on the medical status of patients with type 2 diabetes mellitus: a pilot study. BMC Oral Health 17:77. https://doi.org/10.1186/s12903-017-0369-2

29. Zhao D, Khawaja AT, Jin L et al (2020) Effect of non-surgical periodontal therapy on renal function in chronic kidney disease patients with periodontitis: a systematic review and meta-analysis of interventional studies. Clin Oral Investig 24:1607-1618. https://doi.org/10.1007/s00784-019-03066w

30. Almeida S, Figueredo CM, Lemos C, Bregman R, Fischer RG (2017) Periodontal treatment in patients with chronic kidney disease: a pilot study. J Periodontal Res 52:262-267. https://doi.org/10.1111/jre.12390

31. Glickman I (1971) Periodontal disease. N Engl J Med 284:1071-1077. https://doi.org/10.1056/NEJM197105132841906

32. França LFC, Vasconcelos ACCG, da Silva FRP et al (2017) Periodontitis changes renal structures by oxidative stress and lipid peroxidation. J Clin Periodontol 44:568-576. https://doi.org/10.1111/jcpe.12729

33. Chopra A, Sivaraman K (2019) An update on possible pathogenic mechanisms of periodontal pathogens on renal dysfunction. Crit Rev Microbiol 45:514-538. doi:

10.1080/1040841X.2018.1553847

34. Chen LP, Chiang CK, Chan CP, Hung KY, Huang CS (2006) Does periodontitis reflect inflammation and malnutrition status in hemodialysis patients? Am J Kidney Dis 47:815-822. https://doi.org/10.1053/j.ajkd.2006.01.018

35. Shultis WA, Weil EJ, Looker HC et al (2007) Effect of periodontitis on overt nephropathy and endstage renal disease in type 2 diabetes. Diabetes Care 30:306-311. https://doi.org/10.2337/dc061184

36. Kumar S, Tadakamadla J, Johnson NW (2016) Effect of toothbrushing frequency on incidence and increment of dental caries: a systematic review and meta-Analysis. J Dent Res 95:1230-1236. https://doi.org/10.1177/0022034516655315

37. Magalhães AC, Wiegand A, Rios D, Honório HM, Buzalaf MA (2009) Insights into preventive measures for dental erosion. J Appl Oral Sci 17:75-86. https://doi.org/10.1590/S167877572009000200002

38. Mejia GC, Elani HW, Harper S et al (2018) Socioeconomic status, oral health and dental disease in Australia, Canada, New Zealand and the United States. BMC Oral Health 18:176. 


\section{Tables}

Table1. Baseline participants' characteristics by frequency of daily tooth brushing 
Once a day or not Once to twice a After every $P$ value everyday day meal

$$
(\mathrm{n}=37,522) \quad(\mathrm{n}=5,068) \quad(\mathrm{n}=33,882)
$$

\begin{tabular}{|c|c|c|c|c|c|c|c|}
\hline$\overline{\bar{Y} \text { Year, mean (SD) }}$ & $4 \quad 46.7$ & (12.2) & $4 \quad 42.8$ & "(12.9) & 445.5 & $(12.3)$ & <<0.05 \\
\hline $\begin{array}{l}\text { Follow-up duration, months, } \\
\text { median (IQR) }\end{array}$ & 66.7 & $\begin{array}{l}(25.1- \\
120.6)\end{array}$ & 65.4 & $\begin{array}{l}(31.7- \\
79.9)\end{array}$ & 70.4 & $\begin{array}{l}(26.9- \\
119.6)\end{array}$ & $<0.05$ \\
\hline Male, n (\%) & 23,970 & $(63.9)$ & 2,951 & $(58.2)$ & 11,312 & (33.4) & $<0.05$ \\
\hline Alcohol consumption, $\mathrm{n}(\%)$ & & & & & & & $<0.05$ \\
\hline Abstainer & 13,559 & $(36.1)$ & 2,055 & $(40.6)$ & 14,494 & $(42.8)$ & \\
\hline Occasional drinker & 6,476 & $(17.3)$ & 833 & $(16.4)$ & 6,101 & $(18.0)$ & \\
\hline Regular drinker & 17,487 & $(46.6)$ & 2,180 & $(43.0)$ & 13,287 & $(39.2)$ & \\
\hline Smoking, n (\%) & & & & & & & $<0.05$ \\
\hline Never & 19,571 & $(52.2)$ & 2,968 & $(58.6)$ & 23,826 & $(70.3)$ & \\
\hline Former & 9,423 & $(25.1)$ & 1,215 & $(24.0)$ & 6,351 & $(18.7)$ & \\
\hline Current & 8,528 & $(22.7)$ & 885 & $(17.5)$ & 3,705 & $(10.9)$ & \\
\hline Exercise, n (\%) & & & & & & & $<0.05$ \\
\hline Almost none & 14,762 & $(39.3)$ & 2,024 & $(39.9)$ & 12,144 & $(35.8)$ & \\
\hline 1-2 times a week & 14,100 & $(37.6)$ & 1,871 & $(36.9)$ & 12,415 & $(36.6)$ & \\
\hline 3-5 times a week & 5,387 & $(14.4)$ & 769 & $(15.2)$ & 5,589 & $(16.5)$ & \\
\hline Almost everyday & 3,273 & (8.7) & 404 & $(8.0)$ & 3,734 & $(11.0)$ & \\
\hline$\overline{\text { Body mass index, } \mathrm{n}(\%)}$ & & & & & & & $<0.05$ \\
\hline Underweight & 2,391 & $(6.4)$ & 403 & $(8.0)$ & 4,588 & $(13.5)$ & \\
\hline Normal weight & 25,864 & $(68.9)$ & 3,509 & $(69.2)$ & 24,896 & $(73.5)$ & \\
\hline Obese/ overweight & 9,266 & $(24.7)$ & 1,156 & $(22.8)$ & 4,397 & $(13.0)$ & \\
\hline Medical history, n (\%) & & & & & & & $<0.05$ \\
\hline Any cancer & 1,397 & (3.7) & 160 & $(3.2)$ & 1,351 & $(4.0)$ & \\
\hline Hypertension & 3,265 & (8.7) & 397 & (7.8) & 2,144 & (6.3) & \\
\hline Diabetes & 1,110 & $(3.0)$ & 22 & $(0.4)$ & 588 & (1.7) & \\
\hline
\end{tabular}

Kidney function

\begin{tabular}{lccccccc}
\hline eGFR, mL/min $/ 1.73 \mathrm{~m}^{2}(\mathrm{SD})$ & 80.5 & $(15.1)$ & 82.9 & $(15.1)$ & 82.1 & $(15.5)$ & $<0.05$ \\
\hline CKD risk category, n (\%) & & & & & & & $<0.05$ \\
\hline Low risk & 33,443 & $(89.3)$ & 4,289 & $(85.1)$ & 30,895 & $(91.4)$ & \\
Moderately increased risk & 3,658 & $(9.8)$ & 700 & $(13.9)$ & 2,693 & $(8.0)$ & \\
\hline High risk & 293 & $(0.8)$ & 42 & $(0.8)$ & 191 & $(0.6)$ & \\
\hline Very high risk & 77 & $(0.2)$ & 9 & $(0.2)$ & 41 & $(0.1)$ & \\
\hline
\end{tabular}

CKD stage, $\mathrm{n}(\%)$

$\begin{array}{lcccccc}\text { G1 } & 8,838 & (23.6) & 1,493 & (29.6) & 9,235 & (27.3) \\ \text { G2 } & 26,208 & (69.9) & 3,298 & (65.4) & 22,771 & (67.3) \\ \text { G3a } & 2,260 & (6.0) & 223 & (4.4) & 1,705 & (5.0) \\ \text { G3b } & 144 & (0.4) & 25 & (0.5) & 100 & (0.3) \\ \text { G4 } & 21 & (0.1) & 1 & (0.0) & 9 & (0.0)\end{array}$


${ }^{*}$ Statistically significant $(P$-value of $<0.05)$

Table 2. Comparison of the incidences of composite renal outcome by frequency of daily tooth brushing from multivariate generalized estimating equation. 
Toothbrushing

Once a day or not Reference Reference Reference Reference Reference everyday
$0.28^{*}\left(0.26-0.26^{*}\left(0.24-0.26^{*}\left(0.24-0.26^{*}(0.24-0.26 *(0.24-\right.\right.\right.$

Once to twice a day
$0.30)$
$0.28)$
$0.28)$
$0.28)$
$0.28)$

$0.75^{*}\left(0.71-0.65^{*}\left(0.61-0.64^{*}\left(0.61-0.65^{*}\left(0.62-0.65^{*}(0.62-\right.\right.\right.\right.$

After every meal

$$
0.78
$$

$0.68)$

$0.68)$

$0.69)$

$0.69)$

Year

$1.02^{*}\left(1.01-1.05^{*}\left(1.05-1.05^{*}\left(1.05-1.05^{*}(1.05-1.06(1.06-\right.\right.\right.$

Male $1.02)$ 1.05) $1.05)$ $1.05)$
$0.73 *(0.69-0.82 *(0.78$
$0.86)$
$78-0.83$
* $(0.79$ -
0.8
(0.76-
$0.76)$
$0.88)$
$0.85)$
$0.78(0.74-$
$0.83)$

1.07)

Baseline eGFR,

$\mathrm{mL} / \mathrm{min} / 1.73 \mathrm{~m}^{2}$

$1.05^{*}\left(1.05-1.06^{*}\left(1.06-1.06^{*}\left(1.06-1.06^{*}(1.06-\right.\right.\right.$ $1.05)$ $1.07)$

Alcohol consumption

Abstainers

Reference

Occasional

drinkers

0.94 (0.89 -

NA

Regular drinkers

$0.79 *(0.76-$

0.83 )

Smoking

Never smokers

Reference

Former smokers

0.95 * $(0.90$ -

$0.99)$

Current smokers

$0.96(0.90$ -

1.02)

Exercise

Almost none

\section{Reference}

1-2 times a week

0.99 (0.94 -

1.05)

3-5 times a week

$1.09 *(1.02-$

1.16)

Almost everyday

$1.13^{*}(1.05-$

$1.22)$

Body mass index

Underweight

$1.16 *(1.08-$

1.24)

Normal weight

\section{Reference}

Obese/overweight

$1.12 *(1.06-$

1.18)

Any cancer

$1.27^{*}(1.18-$

1.37)

Hypertension

Diabetes

NA

1.07)

1.07)

$1.06(1.06-$

1.07)

Reference Reference Reference

1.08 * (1.01 - $1.08 *$ (1.01 - 1.09 (1.01 -

$\begin{array}{lll}1.16) & 1.16) & 1.16)\end{array}$

$0.91^{*}\left(0.86-0.91^{*}(0.86-0.9(0.86\right.$ -

$0.95)$

NA

$0.96)$

$0.96)$

Reference Reference Reference

$1.09^{*}(1.02$ - $1.08 *$ (1.02 - 1.07 (1.02 -

$1.15) \quad 1.15)$

$0.95(0.88-0.94(0.87$ 1.02)
1.16)

0.19

\section{NA}


a Model 1 was adjusted for participants' age and sex, baseline eGFR, and baseline chronic kidney disease risk category

b Model 2 was adjusted for health habits (alcohol consumption, cigarette smoking, and exercise habits) in addition to the covariates of Model 1

${ }^{c}$ Model 3 was adjusted for body mass index in addition to the covariates of Model 2 d Model 4 was adjusted for comorbidities (hypertension, diabetes, and any type of cancer) in addition to the covariates of Model 3. Values are odds ratios and adjusted odds ratios (95\% confidence interval)

* Statistically significant $(P$-value of $<0.05)$

Table 3. Risk-based comparison of the incidences of composite renal outcome by frequency of tooth brushing determined from the multivariate generalized estimating equation.

Adjusted odds ratios (95\% confidence interval)

$$
P \text { value }
$$

\begin{tabular}{|c|c|c|c|}
\hline & $\begin{array}{c}\text { Once a day or not } \\
\text { everyday }\end{array}$ & $\begin{array}{l}\text { Once to twice a } \\
\text { day }\end{array}$ & After every meal \\
\hline \multicolumn{4}{|l|}{$\mathrm{CKD}$ risk category ${ }^{\mathrm{a}}$} \\
\hline \multirow[t]{2}{*}{ Low risk } & Reference & $0.21 *(0.19-0.23)$ & $0.63^{*}(0.60-0.67)$ \\
\hline & & $<0.05$ & $<0.05$ \\
\hline \multirow{2}{*}{ risk Moderately increased } & Reference & $0.31^{*}(0.23-0.41)$ & $0.46^{*}(0.35-0.61)$ \\
\hline & & $<0.05$ & $<0.05$ \\
\hline \multirow[t]{2}{*}{ High risk } & Reference & $0.91 \quad(0.46-1.79)$ & $1.11(0.58-2.12)$ \\
\hline & & 0.78 & 0.76 \\
\hline \multirow[t]{2}{*}{ Very high risk } & Reference & $\begin{array}{ll}0.68 & (0.31-1.49)\end{array}$ & $\begin{array}{l}(0.36- \\
1.38)\end{array}$ \\
\hline & & 0.34 & 0.31 \\
\hline
\end{tabular}

${ }^{\text {a }} \mathrm{CKD}=$ chronic kidney disease; model was adjusted for participants' age and sex, baseline eGFR, health habits (alcohol consumption, cigarette smoking, and exercise habits), body mass index, and comorbidities (hypertension, diabetes, and any type of cancer).

* Statistically significant ( $P$-value of $<0.05)$.

\section{Figures}


Figurel. Pationt dispotion

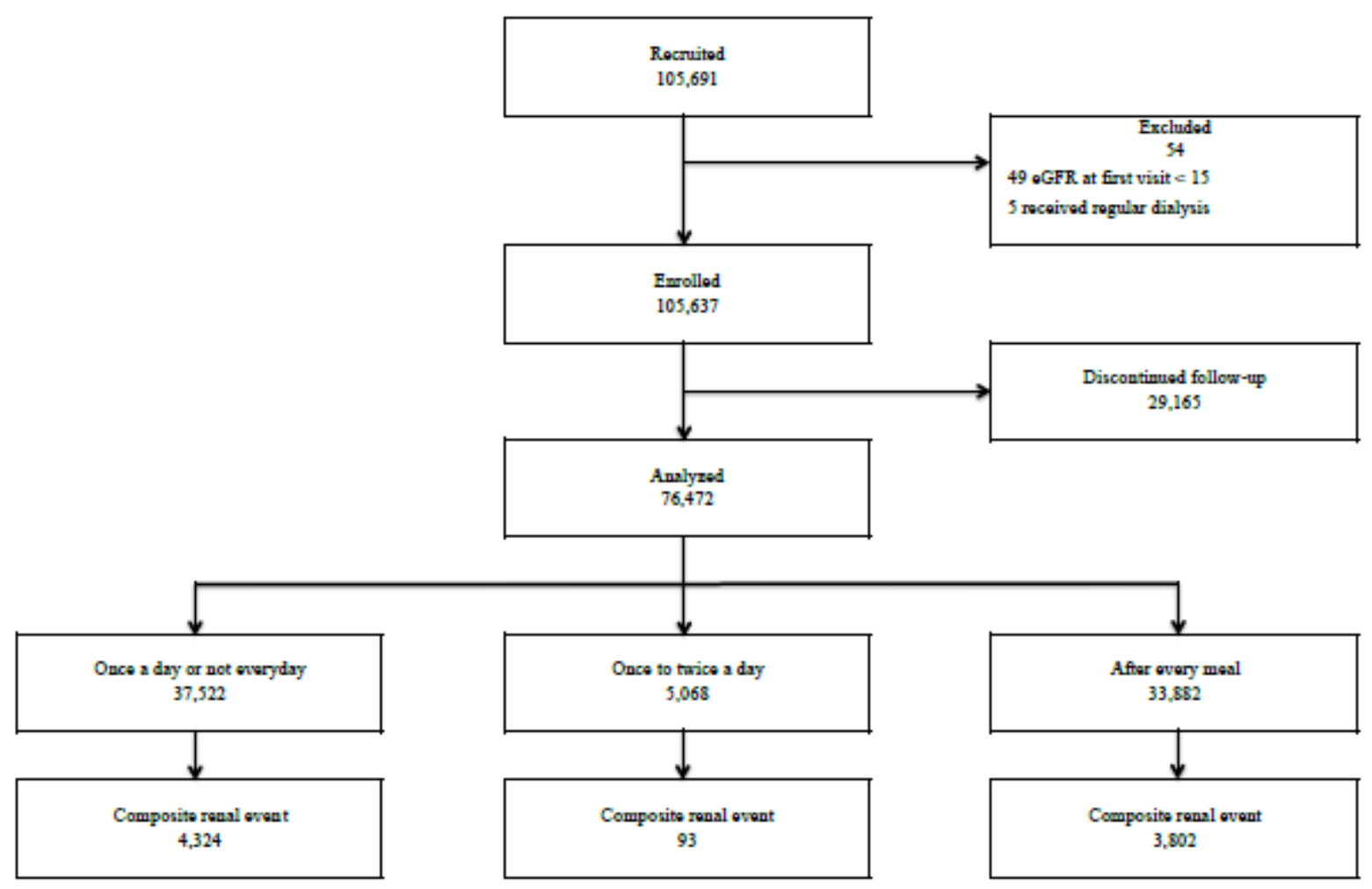

Figure 1

Patient disposition

\section{Supplementary Files}

This is a list of supplementary files associated with this preprint. Click to download.

- visualabstract.jpg 